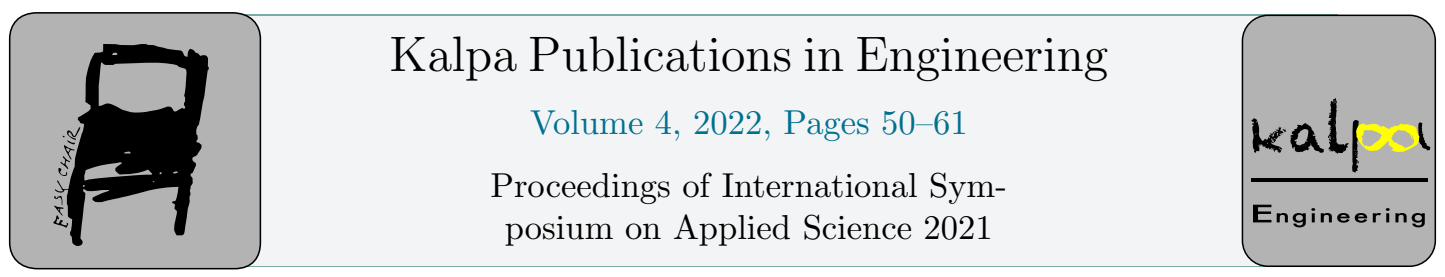

\title{
Study On Some Matrix Equations Involving The Weighted Geometric Mean and Their Application
}

\author{
Xuan Dai Le ${ }^{1,2 *}$ Tuan Cuong Pham ${ }^{1,3}$, Thi Hong Van Nguyen ${ }^{1,2,4}$, Nhat \\ Minh $\operatorname{Tran}^{1,2}$ and Van Vinh Dang ${ }^{1,2}$ \\ ${ }^{1}$ Department of Applied Mathematics, Faculty of Applied Sciences, Ho Chi Minh City \\ University of Technology (HCMUT), 268 Ly Thuong Kiet Street, ward 14, District 10, Ho Chi Minh \\ City, Vietnam \\ ${ }^{2}$ Vietnam National University Ho Chi Minh City, Linh Trung Ward, Thu Duc District, Ho Chi \\ Minh City, Vietnam \\ ${ }^{3}$ Hanoi University of Mining and Geology \\ ${ }^{4}$ University of Finance and Marketing \\ ytkadai@hcmut.edu.vn, nthvan.sdh19@hcmut.edu.vn, \\ nhatminhtccbegmail.com, dangvvinh@hcmut.edu.vn
}

\begin{abstract}
In this paper we consider two matrix equations that involve the weighted geometric mean. We use the fixed point theorem in the cone of positive definite matrices to prove the existence of a unique positive definite solution. In addition, we study the multi-step stationary iterative method for those equations and prove the corresponding convergence. A fidelity measure for quantum states based on the matrix geometric mean is introduced as an application of matrix equation.
\end{abstract}

\section{Introduction}

Let $M_{n}, b \in \mathbb{N}$, be the algebra of $n \times n$ matrices over $C$ and let $P_{n}$ denote the cone of positive definite matrices in $\mathrm{M}_{n}$.

For a real-valued function $f$ and a Hermitian matrix $A \in \mathrm{M}_{n}$ the matrix $f(A)$ is understood by means of the functional calculus.

\footnotetext{
${ }^{*}$ Corresponding author
} 
Let $A, B$ be positive definite matrices. The matrix geometric mean $A \# B=A^{1 / 2}\left(A^{-1 / 2} B A^{-1 / 2}\right) A^{1 / 2}$ was firstly defined by Pusz and Woronowicz [6]. It is the unique positive definite solution of the Riccati equation $X A^{-1} X=B$.

The geometric mean plays an important role in the theory of operator means, operator inequalities and semidefinite programming.

In [5] Lim studied the inverse means problem for the geometric mean and the contraharmonic mean. Using (1) Lim showed that for $\mathrm{A} \leq \mathrm{B}$ the matrix equation $X=A+2 B X^{-1} B$ has a unique solution of the form $X=\frac{1}{2}\left(A+A \#\left(A+4 B A A^{-1}(B)\right)\right.$. In another paper [3], Lim and co-authors studied the nonlinear equation:

$$
X=B \#(A+X)
$$

and showed that this equation has a unique positive definite solution $X=\frac{1}{2}(B+B \#(B+4 A)$. Recently, Lee and co-authors [2] studied the following matrix equation $X^{p}=A+M^{T}(X \# B) M$

They used the Thompson metric and Banach fixed point theorem to show that the equation has a unique positive definite solution. In [8] Zhai and Jin studied two non-linear matrix equations as follows:

and

$$
X^{p}=A+\sum_{i=1}^{m} M_{i}^{T}(X \# B) M_{i}
$$

$$
X^{p}=A+\sum_{i=1}^{j} M_{i}^{T}(X \# B) M_{i}+\sum_{i=j+1}^{m} M_{i}^{T}\left(X^{-1} \# B\right) M_{i}
$$

where $p, m, j$ are positive integers such that $1 \leq j \leq m, A, B$ are positive definite matrices and $M_{i}(i=1,2, \ldots m)$ are nonsingular real matrices

With the Riemannian metric $\delta(A, B)=\left\|\log \left(A^{-1} B\right)\right\|_{2}$ where, $\|X\|_{2}=\left(\operatorname{Tr}\left(X^{2}\right)\right)^{1 / 2}$ for Hermitian matrix $X$, the set $P_{n}$ become a Riemannian manifold. It turns out that the geometric mean $A \# B$ is the midpoint of the geodesic curve $A \#_{t} B=A^{1 / 2}\left(A^{-1 / 2} B A^{-1 / 2}\right)^{t} A^{1 / 2}, \mathrm{t} \in[0,1]$ that joins $\mathrm{A}$ and $\mathrm{B}$.

Motivated by works mentioned above, in this paper we consider a family of non-linear matrix equations:

$$
X^{p}=A+\sum_{i=1}^{m} M_{i}^{T}\left(X \#_{t} B\right) M_{i}
$$

and

$$
X^{p}=A+\sum_{i=1}^{j} M_{i}^{T}\left(X \#_{t} B\right) M_{i}+\sum_{i=j+1}^{m} M_{i}^{T}\left(X^{-1} \#_{t} B\right) M_{i}
$$

where:

$-p, m$ are positive integers

- $A, B$ are $n \times n$ positive definite matrices

- $M_{i}(i=1,2,3, \ldots, n)$ are $n \times n$ non-singular real matrices.

We show that each of these equations has a unique positive definite solution (Theorem 2, Theorem 5). We also study the multi-step stationary iterative method for those equations and prove the corresponding convergence (Theorem 3 and Theorem 6)

Matsumoto's fidelity can also be defined using the well-established notion of the matrix geometric mean. We examine Matsumoto's fidelity through the lens of semidefinite programming to give simple proofs that it possesses many desirable properties for a similarity measure, including monotonicity under quantum channels, joint concavity, and unitary invariance. Finally, we provide a geometric 
interpretation of this fidelity in terms of the Riemannian space of positive definite matrices, and show how this picture can be useful in understanding some of its peculiar properties.

\section{Some Matrix Equations Involving The Weighted Geometric Mean}

Definition 2.1. Let $T: P^{n} \rightarrow P^{n}$ be a operator, we say that $T$ is increasing if $0 \leq x \leq y$ implies $T x \leq T y$.

The following lemma is crucial for us to prove the main results in this paper.

Lemma 1. Let $T: P^{n} \rightarrow P^{n}$ be an increasing operator. Suppose that there exists $r \in(0,1)$ such that $T(s x) \geq s^{r} T(x), \quad x \in P^{n}, \quad s \in(0,1)$

Then $T$ has a unique fixed point $x^{*} \in P^{n}$

Theorem 2. Let $A, B \in P^{n}, m$ be positive integers greater than 2 and $p \geq 1$ Then for nonsingular matrices $M_{1}, M_{1}, \ldots, M_{m}$ in $M_{n}$ the following matrix equation:

$$
X^{p}=A+\sum_{i=1}^{m} M_{i}^{T}\left(X \#_{t} B\right) M_{i}
$$

has a unique positive definite solution $X^{*}$ in $P^{n}$

Proof: Consider the following function

$$
T(X)=\left(A+\sum_{i=1}^{m} M_{i}^{T}\left(X \#_{t} B\right) M_{i}\right)^{\frac{1}{p}}
$$

If we show that the function $T(X)$ is increasing and satisfies the condition of Lemma 1, then it has a unique fixed point $X^{*}$ in $P^{n}$. And hence, the equation (8) has a unique positive definite solution $X^{*}$ in $P^{n}$.

Let $0<X_{1} \leq X_{2}$. According the monotonicity of the weighted geometric mean $\#_{t}$, we have $X_{1} \#_{t} B \leq X_{2} \#_{t} B$. Consequently,

$$
M_{i}^{T}\left(X_{1} \#_{t} B\right) M_{i} \leq M_{i}^{T}\left(X_{2} \#_{t} B\right) M_{i}, \quad i=1,2, \ldots, m
$$

Therefore,

$$
A+\sum_{i=1}^{m} M_{i}^{T}\left(X_{1} \#_{t} B\right) M_{i} \leq A+\sum_{i=1}^{m} M_{i}^{T}\left(X_{2} \#_{t} B\right) M_{i}
$$

Since $p \geq 1$, the function $t^{1 / p}$ is operator monotone on $(0, \infty)$. Then from the last inequality we have:

$$
\begin{aligned}
T(X) & =\left(A+\sum_{i=1}^{m} M_{i}^{T}\left(X \#_{t} B\right) M_{i}\right)^{\frac{1}{p}} \\
& \leq\left(A+\sum_{i=1}^{m} M_{i}^{T}\left(X \#_{t} B\right) M_{i}\right)^{\frac{1}{p}}
\end{aligned}
$$




$$
=T\left(X_{2}\right)
$$

That means, the function $T(X)$ is increasing.

Now, let $X \in P^{n}$. For $t \in(0,1)$ and $p \geq 1$ there exists a constant $r \in(0,1)$ such that $r \leq(1-t) / p$ or $r p+t \geq 1$. It is obvious that for any $s \in(0,1)$, we have $(s X) \#_{t} B=s^{1-t} X \#_{t} B$. Consequently,

$$
M_{i}^{T}\left((s X) \#_{t} B\right) M_{i}=s^{1-t} M_{i}^{T}\left(X \#_{t} B\right) M_{i}, \quad i=1,2, \ldots, m
$$

Since $r p \geq 1-t$, for $s \in(0,1)$, we have $s^{r p} \leq s^{1-t}<1 \in(0,1)$

Therefore,

$$
A+s^{1-t} \sum_{i=1}^{m} M_{i}^{T}\left(X \#_{t} B\right) M_{i} \geq s^{r p}\left(A+\sum_{i=1}^{m} M_{i}^{T}\left(X \#_{t} B\right) M_{i}\right)
$$

On account of operator monotonicity of the function $t^{1 / p}$, from the last inequality we obtain

$$
\begin{aligned}
T(s X)=(A+ & \left.\sum_{i=1}^{m} M_{i}^{T}\left(X \#_{t} B\right) M_{i}\right)^{\frac{1}{p}} \\
\geq & \left(s^{r p}\left(A+\sum_{i=1}^{m} M_{i}^{T}\left(X \#_{t} B\right) M_{i}\right)\right)^{\frac{1}{p}} \\
& =s^{r}\left(A+\sum_{i=1}^{m} M_{i}^{T}\left(X \#_{t} B\right) M_{i}\right)^{\frac{1}{p}}=s^{r} T(X)
\end{aligned}
$$

Thus, the equation (8) has a unique positive definite solution $X^{*}$ in $P^{n}$.

Now, let $X_{1}, X_{2}, \ldots, X_{m} \in P^{n}$ be initial matrices in $P^{n}$. Let us consider the multi-step stationary iterative method for the equation (8)

$$
X_{l+m+1}=\left(A+\sum_{i=1}^{m} M_{i}^{T}\left(X_{l+i} \#_{t} B\right) M_{i}\right)^{\frac{1}{p}}, \quad l=0,1,2, \ldots
$$

In the following theorem we show that the matrix sequence $\left\{X_{k}\right\}$ generated by (5) converges.

Theorem 3. For any $X_{1}, X_{2}, \ldots, X_{m} \in P^{n}$, the matrix sequence $\left\{X_{k}\right\}$ generated by (5) converges to the unique positive definite solution $X^{*}$ of the equation (8)

Proof. For matrices $X_{1}, X_{2}, \ldots, X_{m}$ and $X^{*}$, there exists $a \in(0,1)$ such that

$$
a X^{*} \leq X_{i} \leq a^{-1} X^{*}, \quad i=1,2,3 \ldots m
$$

The strategy of the proof is that we first show that for any $b \in \mathbb{N}$,

$$
a^{r^{b}} X^{*} \leq X_{k} \leq a^{-r^{b}} X^{*}, k=b m+i, \quad(i=1,2,3 \ldots . m)
$$

for some $r \in(0,1)$, with $r \geq \frac{1-t}{p}$. Then, according to the obvious fact that $\lim _{b \rightarrow \infty} a^{r^{b}}=\lim _{b \rightarrow \infty} a^{-r^{b}}=$ 1 and the Squeeze theorem in the normal cone $P^{n}$, it implies that $\left\{X_{k}\right\}$ converges to $X^{*}$.

Now we prove (7) using the method of mathematical induction. For $b=0$, the inequality (7) reduces to the case of (6). Assume that (7) is true for $b=q-1$ for some positive integer $q$, i.e., for $k=$ $(q-1) m+i,(i=1,2,3, \ldots, m)$, that is,

$$
a^{r^{q-1}} X^{*} \leq X_{(q-1) m+i} \leq a^{-r^{q-1}} X^{*} .
$$


Mention that, $X_{l+m+1}=T\left(X_{(q-1) m+i}\right)$. From the proof of Theorem 2, the map $T(X)$ is increasing. Therefore, from (8) it implies that:

$$
T\left(a^{r^{q-1}} X^{*}\right) \leq X_{q m+i}=T\left(X_{(q-1) m+i}\right) \leq T\left(a^{-r^{q-1}} X^{*}\right) .
$$

From (4) we have:

$$
T\left(a^{r^{q-1}} X^{*}\right) \geq a^{r^{b}} T\left(X^{*}\right)=a^{r^{b}} X^{*} .
$$

On the other hands, since $a^{-r^{q-1}} \geq 1$ the inequality (3) is reversed, hence, (4) is also reversed. Again using the monotonicity of $T(X)$ we have:

$$
T\left(a^{r^{q-1}} X^{*}\right) \leq a^{-r^{b}} T\left(X^{*}\right)=a^{-r^{b}} X^{*} .
$$

From (9) and (10), it implies that

$$
a^{r^{q-1}} X^{*} \leq X_{q m+i} \leq a^{-r^{b}} X^{*}
$$

Thus, (7) is true, and $\left\{X_{k}\right\}$ converges to $X^{*}$.

Definition 2.2: Suppose that $D \subset E$, then $D \times D \subset E \times E$. Let $T: D \times D \rightarrow E$ be a operator, we say that $T$ is mixed monotone if for any $x_{1}, x_{2}, y_{1}, y_{2} \in D$ with $x_{1} \leq x_{2}, y_{1} \geq y_{2} \in D$ implies $T\left(x_{1}, y_{1}\right) \leq T\left(x_{2}, y_{2}\right)$. And $x^{*}$ is a fixed point of $T$ if it satisfies $x^{*}=T\left(x^{*}, x^{*}\right), x^{*} \in D$

Lemma 4: Let T: $P^{n} \times P^{n} \rightarrow P^{n}$ be a mixed monotone operator. Suppose that for any $0<a<b<$ 1 , there exists a constant $\beta=\beta(a, b) \in(0,1)$ such that $T\left(t x, \frac{1}{t} x\right) \geq t^{\beta} T(x, x), \quad x \in P^{n}, \quad t \in(0,1)$.

Then $T$ has a unique fixed point $x^{*} \in P^{n}$

Theorem 5: Let $A, B \in P^{n}$ and $M_{i}(i=1,2, \ldots, n)$ be nonsingular matrices in $M_{n}$ Let $m$ be positive integers greater than $2, p \geq 1$, and $j$ be some integer between 1 and $m$. Then the nonlinear matrix equation

$$
X^{p}=A+\sum_{i=1}^{j} M_{i}^{T}\left(X \#_{t} B\right) M_{i}+\sum_{i=j+1}^{m} M_{i}^{T}\left(X^{-1} \#_{t} B\right) M_{i}
$$

has a unique positive definite solution $X^{*}$ in $P^{n}$.

Proof: Consider the following operator

$$
T(X, Y)=\left(A+\sum_{i=1}^{j} M_{i}^{T}\left(X \#_{t} B\right) M_{i}+\sum_{i=j+1}^{m} M_{i}^{T}\left(X^{-1} \#_{t} B\right) M_{i}\right)^{\frac{1}{p}}
$$

Obviously, $T: P^{n} \times P^{n} \rightarrow P^{n}$. If we show that the operator $T$ satisfies the condition of Lemma 4 , then $T$ has a unique fixed point $X^{*} \in P^{n}$. Therefore, the equation (11) has a unique positive definite solution $X^{*} \in P^{n}$.

Firstly mention that according to the monotonicity of the weighted geometric mean, the function $T^{p}(X, Y)$ is increasingly monotone in $X$ and decreasingly monotone in $Y$. Therefore, $T^{p}(X, Y)$ is a mixed monotone map. For $p \geq 1$, the function $t^{1 / p}$ is operator monotone on $(0, \infty)$. Thus, the function $T(X, Y)=\left(T^{p}(X, Y)\right)^{\frac{1}{p}}$ is also mixed monotone.

Secondly, for $t \in(0,1)$ and $p \geq 1$ there exists a constant $r \in(0,1)$ such that $r \leq(1-t) / p$ or $r p+t \geq 1$. Let $X \in P^{n}$, on account of the operator monotonicity of the function $t^{1 / p}$, we have 


$$
\text { (13) } \begin{aligned}
T\left(s X, s^{-1} X\right) & =\left(A+\sum_{i=1}^{j} M_{i}^{T}\left((s X) \#_{t} B\right) M_{i}+\sum_{i=j+1}^{m} M_{i}^{T}\left(\left(s^{-1} X\right)^{-1} \#_{t} B\right) M_{i}\right)^{\frac{1}{p}} \\
& =\left(A+\sum_{i=1}^{j} M_{i}^{T}\left((s X) \#_{t} B\right) M_{i}+\sum_{i=j+1}^{m} M_{i}^{T}\left(\left(s X^{-1}\right) \#_{t} B\right) M_{i}\right)^{\frac{1}{p}} \\
\geq & \left(s^{r p}\left(A+\sum_{i=1}^{j} M_{i}^{T}\left((s X) \#_{t} B\right) M_{i}+\sum_{i=j+1}^{m} M_{i}^{T}\left(\left(s X^{-1}\right) \#_{t} B\right) M_{i}\right)\right)^{\frac{1}{p}} \\
= & s^{r}\left(A+\sum_{i=1}^{j} M_{i}^{T}\left((s X) \#_{t} B\right) M_{i}+\sum_{i=j+1}^{m} M_{i}^{T}\left(\left(s X^{-1}\right) \#_{t} B\right) M_{i}\right)^{\frac{1}{p}} \\
& =s^{r} T(X, X)
\end{aligned}
$$

Thus, the equation (11) has a unique positive definite solution $X^{*}$ in $P^{n}$ as the map $T(X, Y)$ satisfies all hypotheses of Lemma 4 .

Similar to the case of (5) we may use the following multi-step stationary iterative method to find the solution of (11):

$$
\text { (14) } X_{l+m+1}=\left(A+\sum_{i=1}^{j} M_{i}^{T}\left(X_{l+i} \#_{t} B\right) M_{i}+\sum_{i=j+1}^{m} M_{i}^{T}\left(X_{l+i}^{-1} \#_{t} B\right) M_{i}\right)^{\frac{1}{p}}, l \in \mathbb{N}
$$

where $X_{1}, X_{2}, \ldots, X_{m} \in P^{n}$ are initial matrices.

The proof of the following theorem is similar to the one of Theorem 3.

Theorem 6. For any $X_{1}, X_{2}, \ldots, X_{m} \in P^{n}$, the matrix sequence $\left\{X_{k}\right\}$ generated by (3) converges to the unique positive definite solution $X^{*}$ của phương trình (11).

Proof: Since equation (11) has a unique positive definite solution $X^{*}$, there exists a positive constant $0<a<1$ such that the initial matrices $X_{1}, X_{2}, \ldots, X_{m} \in P^{n}$ satisfy

$$
a X^{*} \leq X_{i} \leq a^{-1} X^{*}, \quad i=1,2,3, \ldots, m
$$

We first show that for any $b \in \mathbb{N}$,

$$
a^{r^{b}} X^{*} \leq X_{i} \leq a^{-r^{b}} X^{*} k=b m+i(i=1,2,3, \ldots, m)
$$

For some $t \in(0,1)$ with $r \geq \frac{1-t}{p}$. Then, according to the obvious fact that $\lim _{b \rightarrow \infty} a^{r^{b}}=\lim _{b \rightarrow \infty} a^{-r^{b}}=1$ and the Squeeze theorem in the normal cone $P^{n}$ it implies that $\left\{X_{k}\right\}$ converges to $X^{*}$.

Now we prove (16) using the method of mathematical induction. For $b=0$, the inequality (16) reduces to the case of (15). Assume that (16) is true for $b=q-1$, for some positive integer $q$ i.e., for $k=(q-1) m+i,(i=1,2,3, \ldots, m)$ that is,

$$
a^{r^{q-1}} X^{*} \leq X_{(q-1) m+i} \leq a^{-r^{q-1}} X^{*}
$$

Mention that, $X_{q m+i}=T\left(X_{(q-1) m+i}, X_{(q-1) m+i}\right)$. From the proof of Theorem 5, the map $T$ is mixed monotone. Therefore, from (17) it implies that 


$$
T\left(a^{r^{q-1}} X^{*}, a^{-r^{q-1}} X^{*}\right) \leq X_{q m+i}=T\left(X_{(q-1) m+i}, X_{(q-1) m+i}\right) \leq T\left(a^{r^{q-1}} X^{*}, a^{-r^{q-1}} X^{*}\right)
$$

From (13) we have

$$
T\left(a^{r^{q-1}} X^{*}, a^{-r^{q-1}} X^{*}\right) \geq a^{r^{q}} T\left(X^{*}, X^{*}\right)=a^{r^{q}} X^{*}
$$

On the other hands, since $a^{-r^{q-1}} \geq 1$ the inequality (13) is reversed. Again using the mixed monotonicity of $T$ we have

$$
T\left(a^{-r^{q-1}} X^{*}, a^{r^{q-1}} X^{*}\right) \leq a^{-r^{q}} T\left(X^{*}, X^{*}\right)=a^{-r^{q}} X^{*}
$$

From (18) and (19), it implies that

$$
a^{r^{q}} X^{*} \leq X_{q m+i} \leq a^{-r^{q}} X^{*}
$$

Thus, (16) is true, and $\left\{X_{k}\right\}$ converges to $X^{*}$.

\section{Application}

Matsumoto fidelity after its introduction by Matsumoto in [12], which is defined as:

$$
F_{M}(\rho, \sigma):=\operatorname{Tr}(\rho \# \sigma), \rho \# \sigma=\rho^{1 / 2}\left(\rho^{1 / 2} \sigma^{1 / 2} \rho^{1 / 2}\right) \rho^{1 / 2}
$$

for invertible quantum states $\rho$ and $\sigma$. The binary operation \# is known as the matrix weighted

geometric mean with $t=\frac{1}{2}$, it is also the geometric mean. The matrix geometric mean has intricate connections to the geometry of quantum state space and also to quantum information theory.

$F_{M}(\rho, \sigma)$ is the matrix equation involving the weighted geometric mean.

Semidefinite programming is a well-behaved class of optimization problems which have seen countless applications in the study of quantum theory, including convex geometry, thermodynamics, computational complexity theory, cryptography, Bell non-locality, and entanglement, to just name a few. Fortunately, the Matsumoto fidelity can be formulated as a semidefinite program (abbreviated as SDP) which allows a convenient prescription for its calculation, and also provides a useful analytical definition with which many of its properties can be easily proven.

\subsection{Geometric interpretation}

The space of positive definite matrices can be pictured as a cone like the one shown in Figure 1 


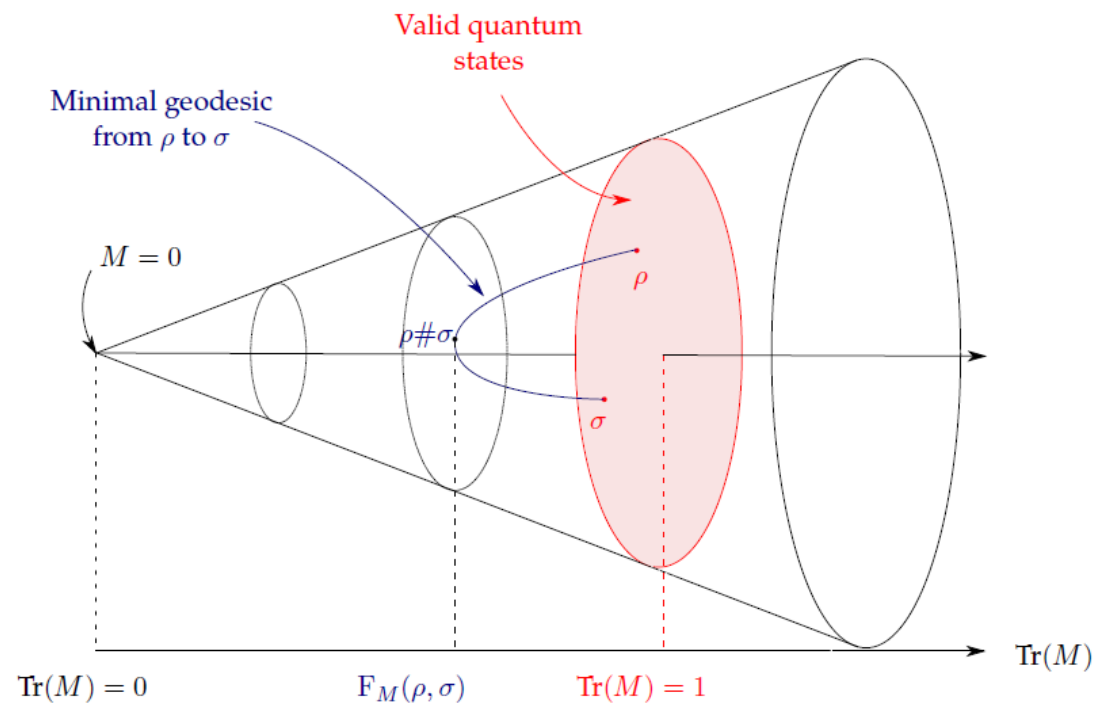

Figure 1: The space of positive semidefinite matrices

The space of positive semidefinite matrices is presented pictorially as a cone with boundary, embedded into the ambient space of Hermitian $n \times n$ matrices. The positive definite matrices form the interior of the cone, whereas singular matrices form the boundary (because an infinitesmal perturbation can change a zero eigenvalue to negative, putting it outside the cone). The central axis represents scalar multiples of the identity, such as the maximally mixed state. The distance measure in Equation (20) defines geodesics (i.e. shortest-length paths) within this conical space. These geodesics always curve toward lower trace (leftward in the picture). The Matsumoto fidelity, denoted by $F_{M}$ - the trace of the midpoint of this geodesic - lies between 0 and 1 , and measures the "closeness" between states according to how far leftward the geodesic curves.

This space has a unique invariant Riemannian metric, with the metric tensor $g$ defined at a particular point (i.e. matrix) $M$ by:

$$
\left.g(\rho, \sigma)\right|_{M}=\operatorname{Tr}\left(M^{-1} \rho M^{-1} \sigma\right)
$$

$\rho, \sigma, M$ are positive definite matrices.

As shown in Figure 1, this geodesic curves towards the tip of the cone (the 0 matrix), and the Matsumoto fidelity is a measure of how far it curves (i.e. how small the trace of the midpoint becomes). Quantum states that are close together (with respect to this metric) in the space of all quantum states have a geodesic which does not deviate far from that space, and so the trace of the midpoint is close to unity.

\subsection{Qubits}

The metric in Equation (20) takes a particularly simple form for qubits, using the following parameterization for positive definite $2 \times 2$ matrices.

$$
\rho(\alpha, r, \theta, \phi)=e^{i \phi \sigma_{z}} e^{i \phi \sigma_{y}}\left(\begin{array}{cc}
e^{-\frac{\alpha+r}{\sqrt{2}}} & 0 \\
0 & e^{-\frac{\alpha+r}{\sqrt{2}}}
\end{array}\right) e^{-i \phi \sigma_{z}} e^{-i \phi \sigma_{y}}
$$


Where $\alpha, r, \theta, \phi \in \sim$

Then it can be shown that the metric in Equation (20) becomes:

$$
\begin{aligned}
d \mathrm{~s}^{2} & =\operatorname{Tr}\left(\rho^{-1} d \rho \rho^{-1} d \rho\right) \\
& =d \alpha^{2}+d \mathrm{r}^{2}+\sinh ^{2} r\left(d \theta^{2}+\sin ^{2} \theta d \phi^{2}\right)
\end{aligned}
$$

The metric for $(r, \theta, \phi)$ can be recognized as three-dimensional hyperbolic space in radial coordinates, meaning that the geometry of $2 \times 2$ positive definite matrices with this metric is $\mathbb{R} \times \mathbb{H}_{3}$.

Now $r$ can be understood as parameterizing the purity of the state; with $r \rightarrow \infty$ for a pure state and $r=0$ for the maximally mixed state. The parameter $\alpha$ is fixed for a quantum state once $\mathrm{r}$ is determined due to the unit trace condition, as the trace of $\rho(\alpha, r, \theta, \phi)$ is given by:

$$
\operatorname{Tr}(\rho(\alpha, r, \theta, \phi))=2 e^{-\frac{\alpha}{\sqrt{2}}} \cosh \left(\frac{r}{\sqrt{2}}\right)
$$

For quantum states, we have $\alpha=\alpha_{q}(r):=-\sqrt{2} \log \left(\frac{1}{2} \cosh \left(\frac{r}{\sqrt{2}}\right)\right)$ so that $\rho\left(\alpha_{q}(r), r, \theta, \phi\right)$ has trace 1 . The angular coordinates $\theta$ và $\phi$ are analogous to the angular coordinates of the Bloch sphere (Figure 2)

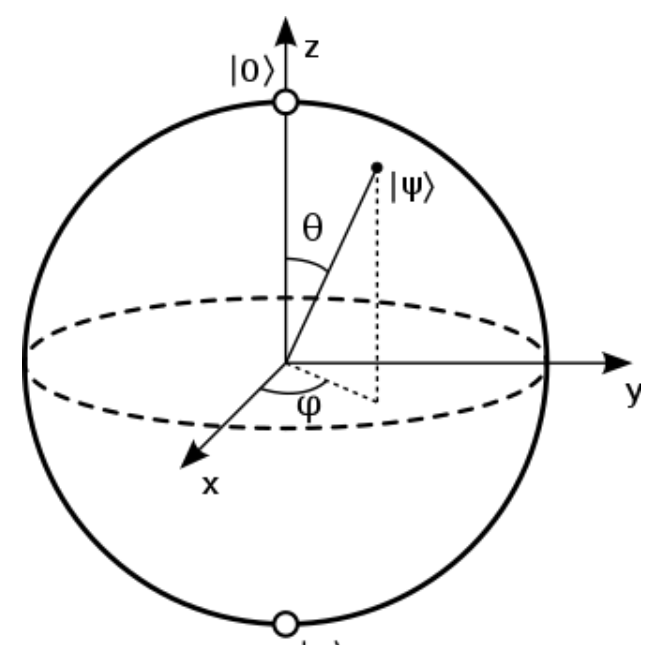

$|1\rangle$

Figure 2: Block Sphere

Effectively, the $\sinh ^{2} r$ prefactor in front of the coordinates in the metric means that a curve is always shorter if it bends "inwards" towards lower $r$. A result of this is that the geodesic 


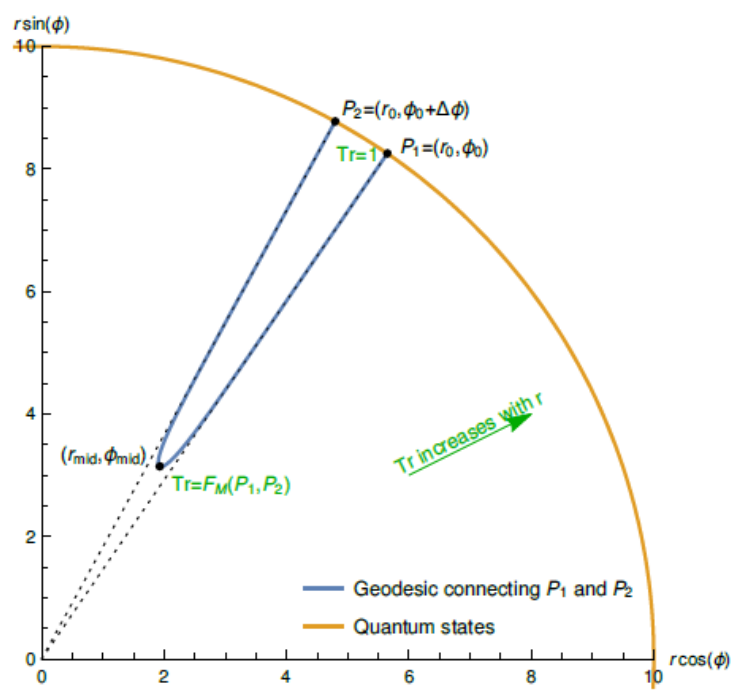

Figure 3: An example of the geometric interpretation of the Matsumoto fidelity

This is for the example discussed in the text: two qubit states with the same purity (characterized by the radial coordinate $r$ ) and angular coordinate $\phi$ (representing the angular coordinate separating them on the Bloch sphere). However, unlike the Bloch sphere, only the orange line (at fixed $r=r_{0}$ represents valid quantum states with trace 1 ; all other points in the plot are $2 \times 2$ positive definite matrices with non-unit trace. The space is associated with a hyperbolic geometry, so that the minimal geodesic between the points labelled $\rho_{1}$ and $\rho_{2}$ (shown in blue) is not a straight line, but rather bends inwards. The trace at the midpoint along the geodesic (i.e. where $r=r_{\text {mid }}$ is the Matsumoto fidelity, and scales as $\exp \left(r_{\text {mid }}-r_{0}\right)$. As the states approach purity, $r_{0}$ diverges to infinity, and $r_{\text {mid }}$ remains fixed as explained in the text, so the trace goes to zero regardless of how small $\Delta \phi$ is. This plot was made using $r_{0}=10, \Delta \phi=0.1$, and the equation for the geodesic connecting the two points (with $\phi_{0}=0$ for convenience) is parameterized by the equation $r(\phi)=\operatorname{arctanh}\left(\frac{\tanh \left(r_{0}\right)}{\cos (\phi)-\frac{\tanh \left(r_{0}\right)}{\frac{\sin (\phi)(\cos (\Delta \phi-1)}{\sin (\Delta \phi)}}}\right)$ between two quantum states passes through states with small siner $r$ but the same $\alpha$ and hence has trace less than 1 - i.e. the Matsumoto fidelity is less than 1 (see Figure 2).

Let us use this geometrical picture to understand why the Matsumoto fidelity of two almost identical pure states is 0 . Suppose we have two nearly-pure states with identical $r=r_{0}$ (which we eventually take to infinity so that the states become pure), and $\phi$ differing by a fixed (arbitrarily small) $\Delta \phi$. Fix $\alpha=\alpha_{q}\left(r_{0}\right)$ and $\phi=\frac{\pi}{2}$ for simplicity. With these constraints, the geodesic between the states is restricted to a two-dimensional subspace parametrized by $r$ and $\phi$, with the reduced metric

$$
d s^{2}=d r^{2}+\sinh ^{2} r d \phi^{2}
$$

This is exactly the radial coordinates for the hyperbolic plane $\mathbb{H}_{2}$

To determine the Matsumoto fidelity of these two states, we need to find the trace of the midpoint of the geodesic connecting them in this space. Solving the geodesic equation gives the curve shown in Figure 3, which curves inwards toward the center. One can show that the midpoint $\left(r_{\text {mid }}, \phi_{\text {mid }}\right)$ of the geodesic is at $r_{\text {mid }}=\arctan h\left(\tanh \left(r_{0}\right)\right) \cos \left(\frac{\Delta \phi}{2}\right)$ and $\phi_{\text {mid }}=\phi_{0}+\frac{1}{2} \Delta \phi \$$. 
For large $r_{0}$, the former goes as

$$
r_{\text {mid }}=\arctan h \cos \frac{\Delta \phi}{2}+O\left(e^{-2 r_{0}}\right)
$$

So for large $r_{0}, r_{\text {mid }}$, becomes independent of $r_{0}$. This means that the minimum radius $r_{\text {mid }}$ of the geodesic shown in Figure 3 remains fixed even as $r_{0} \rightarrow \infty$.

Now let us evaluate the trace of this midpoint in order to determine the Matsumoto fidelity. On this subspace, and at large $r_{0}$, the trace is

$$
\operatorname{Tr}\left(\rho\left(\alpha_{q}\left(r_{0}\right), r, \frac{\pi}{2}, \phi\right)\right)=\frac{\cosh (r / \sqrt{2})}{\cosh \left(r_{0} / \sqrt{2}\right)}=\frac{1}{2} e^{-\frac{r_{0}}{\sqrt{2}}} \cosh \left(\frac{r}{\sqrt{2}}\right)+O\left(e^{-\sqrt{2} r_{0}}\right)
$$

Thus the Matsumoto fidelity of these two states is:

$$
F_{M}\left(\rho\left(\alpha_{q}\left(r_{0}\right), r, \frac{\pi}{2}, \phi\right), \rho\left(\alpha_{q}\left(r_{0}\right), r_{0}, \frac{\pi}{2}, \phi_{0}+\Delta \phi\right)\right)=f(\Delta \phi) e^{-\frac{r_{0}}{\sqrt{2}}}+O\left(e^{-\sqrt{2} r_{0}}\right)
$$

with $f(\Delta \phi)=\frac{1}{2} \cosh \left(\frac{1}{\sqrt{2}} \arctan h \frac{\Delta \phi}{2}\right)$ independent of $r_{0}$. Thus for fixed $\Delta \phi$ and arbitrarily large $r_{0}$, we can see that this goes to 0 , demonstrating that the Matsumoto fidelity of two distinct pure states is 0 . We can also see why it goes to 0 so slowly when states are almost pure and almost identical, as shown in the top-right diagram of Figure 1; one can verify that $\mathrm{f}(\Delta \phi)$ diverges to infinity as $\Delta \phi \rightarrow 0$ meaning that $r_{0}$ needs to become very large to suppress this prefactor i.e. the states need to be "almost pure" before the strange behaviour of similar states having negligible fidelity occurs. A similar argument to the one presented here can be constructed for non-qubit states. This example demonstrates how the geometric picture can be useful in understanding the behavior of the Matsumoto fidelity.

\section{Conclusion}

In this paper, we have explored the matrix equation involving the weighted geometric mean and their application to the Matsumoto fidelity for quantum states. It is also motivated by connecting to the geometry of positive definite matrices. The others matrix equations and their uses will be the subject of future paper.

\section{Conflicts of Interest}

The authors declare no conflicts of interest.

\section{Acknowledgment}

We acknowledge the support of time and facilities from Ho Chi Minh City University of Technology (HCMUT) - VNU-HCM for this study.

\section{References}

[1] D.Guo, V.Lakshmikantham (1988). Nonlinear Problems in AbstractCones 
[2] C.Jung, H.M. Kim, Lim (2009), "On the solution of the nonlinear matrix equation $\mathrm{X}^{\mathrm{n}}=\mathrm{f}(\mathrm{X})$ ”, Linear Algebra Appl. 430, 2042-2052

[3] Y. Lim (2005), "Inverse mean problem of geometric and contraharmonic means".

[4] W. Pusz, S.L.Woronowicz (1975), "Functional calculus for sesquilinear forms and the purification map\}, Vol.8, No.2, 159-170

[5] C.Jung, H.M. Kim, Lim (2009) “On the solution of the nonlinear matrix equation $\$ X^{\wedge} n=f(X) \$$ ”, Linear Algebra Appl. 430, 2042-2052

[6] Sam Cree, Jamie Sikora (2020), “A fidelity measure for quantum states based on the matrix geometric mean"

[7] C.Zhai, Z.Jin (2020), "Solvability for two forms of nonlinear matrix equations", Bull. Iran. Math. Soc

[8] Armin Uhlmann (2011), "Transition Probability (Fidelity) and its Relatives" 